\title{
Politique
}

\section{La notion de discipline scientifique}

\section{Michel Leclerc}

Numéro 15, hiver 1989

Paradigmes et scientificité

URI : https://id.erudit.org/iderudit/040618ar

DOI : https://doi.org/10.7202/040618ar

Aller au sommaire du numéro

Éditeur(s)

Société québécoise de science politique

ISSN

0711-608X (imprimé)

1918-6584 (numérique)

Découvrir la revue

Citer cet article

Leclerc, M. (1989). La notion de discipline scientifique. Politique, (15), 23-51.

https://doi.org/10.7202/040618ar d'utilisation que vous pouvez consulter en ligne.

https://apropos.erudit.org/fr/usagers/politique-dutilisation/ 


\title{
La notion de discipline scientifique et ses enjeux sociaux
}

\author{
Michel Leclerc \\ École nationale d'administration publique
}

La notion de discipline scientifique, parce qu'elle repose sur un paradoxe, échappe à toute définition précise. Au seuil de cet essai, il importe en effet d'affirmer que la discipline ne peut être univoquement définie. Certes celle-ci renvoie d'abord à un ensemble d'unités discursives dont la configuration particulière délimite une aire autonome de recherche et d'expérimentation.

Mais la discipline est aussi un mécanisme social de différenciation intellectuelle. Elle est également une micro-institution, au sens que Durkheim attribuait à ce terme, c'est-à-dire un instrument de contrôle social dont relève la régulation idéologique des activités scientifiques. On entend donc ici par discipline un mode de connaissance systématique qui se réalise à travers la diversité des enjeux sociaux institutionnalisés.

Aussi bien, la discipline ne peut plus être pensée comme une figure fermée sur elle-même, hermétiquement close, et dont les limites rationnelles seraient fixées ne varietur. Contre une telle vision positiviste de la discipline, il convient d'opposer une conception qui 
met en évidence le relativisme et la précarité des interactions sociales à l'oeuvre dans la discipline. Il n'existe pas d'hétérogénéité radicale entre la discipline définie comme construction discursive et la discipline considérée comme manifestation rationnelle d'enjeux sociaux organisés. L'une et l'autre, en effet, ne s'excluent pas dans la réalité.

Nous voulons précisément montrer que la succession de l'une à l'autre ne peut être comprise sans référence à l'identification des enjeux sociaux qui structurent la discipline. À cette fin, notre démarche empruntera les étapes suivantes.

Dans sa première partie, cet article retracera sommairement quelques-uns des principaux moments de l'évolution sémantique de la notion de discipline. Dans la deuxième partie, nous examinerons le rôle de la philosophie positiviste dans la constitution d'un objet disciplinaire. Nous tenterons notamment d'illustrer de quelle manière le projet unificateur et dogmatique d'Auguste Comte a contribué à paralyser la réflexion sur le «système de la science», mais aussi à fournir des arguments épistémologiques aux luttes disciplinaires pour la maîtrise des biens sociaux rattachés à la pratique scientifique. Une troisième partie montrera comment la différenciation disciplinaire est une prémisse nécessaire à toute volonté de monopolisation de l'autorité scientifique. Dans la dernière partie enfin, nous verrons en quoi l'habitus disciplinaire, en tant que mode de diffusion sociale de l'identité professionnelle, perpétue les rapports d'autorité et de contrôle institutionnels au sein de la discipline, légitime les luttes à l'intérieur du champ scientifique et détermine au bout du compte la pratique et le contenu de la discipline.

\section{Origines sémantiques de la sémantique}

C'est la Chanson de Roland en 1080 qui introduit pour la première fois dans le vocabulaire français le mot discipline. Sous ce vocable emprunté au latin classique, se superposent sous la forme de strates sémantiques successives l'action d'apprendre, la matière d'enseignement ainsi que le système philosophique transmis. Le latin ecclésiastique donne à ce mot un sens fort différent et très éloigné de 
l'idée originale. Peu à peu les règles monastiques imposeront la notion de «correction» et de «châtiment» corporel ${ }^{1}$. C'est d'ailleurs cette définition qui prévaudra au XVIe siècle, allant même jusqu'à désigner l'expérience du «massacre» et du «carnage» ${ }^{2}$. L'ancien et le moyen français conféreront un sens encore plus restrictif à ce mot, la discipline servant alors non plus à identifier la punition corporelle mais l'instrument de la mortification imposée par la règle. C'est ainsi que dans la Comédie Mont-de-Marsan, Marguerite de Navarre décrira la «discipline» comme le «fouet servant à la flagellation» 3 .

Mais il fraudra attendre la Renaissance pour que la discipline caractérise enfin un corps de savoir transmissible selon les «règles de l'enseignement imposées par un maître à ceux qu'il veut former ${ }^{4}$. Il est à noter que pendant la majeure partie du Moyen-Age les idées d'apprentissage, d'éducation ou de formation étaient restées fortement indexées à un sentiment de prescription autoritaire. Le disciple (l'«élève» selon le latin classique) est ainsi assujetti aux règles de l'inftruction, du «châtiment volontaire, ou enjoint par pénitence» 5 , c'est-à-dire soumis à une contrainte qui encerclait simultanément le corps et la raison. De telle sorte que le système de la discipline, tel qu'il s'imposera au XIXe siècle comme modèle général de transmission du savoir, appartient historiquement à ces «schémas de docilité» décrit par Michel Foucault dans Surveiller et punir et dont le Siècle des Lumières avait déjà repéré les premiers signes encore imperceptibles. CNRS, 1979.

1. Trésors de la langue française, Tome VII, Paris, Éditions du

2. Oscar Bloch, W. von Wartburg, Dictionnaire étymologique de la langue française, Paris, PUF, 1964, p. 196.

3. Trésors..., idem.

4. Walter von Wartburg, Franzosisches Etymologisches Worterbuch, Band III, Tübingen, J.C.B. Mohr (Paul Siebeck), 1949, p. 92. Dans l'oeuvre de Nicolas Oresme pourtant, dès la fin du XIVe siècle, la discipline est entendue au sens de la règle (disciplina) commune à un ensemble de discours pédagogiques codifiés par l'École ou l'Université à des fins d'enseignement (discere). (J.-P. Resweber, La méthode interdisciplinaire, Paris, PUF, 1981, pp. 43-51.)

5. Gilles Ménage, Dictionnaire étymologique de la langue française, (1750), Tome I, Genève, Slatkine Reprints, 1972, p. 479. 
Procédures de domination physique à l'origine, les disciplines seront décrites, à partir du XVIIIe siècle, comme des «méthodes qui permettent le contrôle minutieux des opérations du corps qui assurent l'assujettissement constant de ses forces et leur imposent un rapport de docilité-utilité» ${ }^{6}$.

À partir du XVIIIe siècle, la coercition disciplinaire ${ }^{7}$, exclut le rituel du renoncement, amplifiant par contraste les opérations du corps dans la minutie de ses techniques d'examen, de contrôle et de domination soupçonneuse. Ce processus coïncide avec sa transposition au domaine du savoir puisqu'on peut aisément affirmer que l'émergence des sciences humaines résulte de l'extension du modèle disciplinaire aux éléments constitutifs du discours savant. En effet, comme l'écrit Michel Foucault, «ces sciences dont notre «humanité» s'enchante depuis plus d'un siècle ont leur matrice technique dans la minutie tatillonne et méchante des disciplines et de leurs investigations» ${ }^{8}$.

L'instance disciplinaire introduit ainsi un protocole de contrainte à partir duquel s'instaure une division rigide entre le sujet soumis (à la sanction scolaire, à l'inquisition pénitentiaire, à l'ascétisme monastique) et le surveillant, reproduisant ainsi au passage «le schéma du pouvoir-savoir propre à toute discipline»9. Bref, la discipline est d'abord un type de pouvoir sanctionné par des institutions qui se l'approprient à leurs fins dans un système mixte d'exclusion et de domination. L'Encyclopédie n'a-t-elle pas d'ailleurs mis au jour cette rencontre objective du savoir et de l'autorité en associant la «discipline» à l'idée charnière de gouvernement ${ }^{10}$. Ainsi, dans le champ particulier du pouvoir le mécanisme de la discipline

6. Michel Foucault, Surveiller et punir, Naissance de la prison, Paris, 1975, Éditions Gallimard, p. 139.

7. Signalons que le qualificatif «disciplinaire» n'apparaît qu'en 1611 seulement et que son usage reste exceptionnel jusqu'au XIXe siècle (Albert Dauzat, Jean Dubod, Henri Mitterand, Nouveau dictionnaire étymologique et historique, Paris, Librairie Larousse, 1971, p. 238.

8. M. Foucautl, op. cit., p. 227.

9. Ibid., p. 228.

10. Encyclopédie ou Dictionnaire raisonnée des sciences, des arts et des métiers, par une société de gens de lettres, (1751), Vol. 1, Tome I-XI, N.Y., Readex Microprints Corporation, 1969, p. 972. 
délimite des espaces clos: la caserne, l'usine, l'école, le couvent. De même, transposée dans l'univers du savoir, la technique disciplinaire préside à une partition générale des connaissances qui détermine l'apparition d'une instance inédite: celle des disciplines scientifiques.

\section{La partition positiviste des disciplines}

La découpe des savoirs scientifiques opérée par Auguste Comte au milieu du XIXe siècle pose de façon systématique le problème de la spécificité épistémologique des sciences. On sait que chez Comte la pluriélisation du savoir obéit à un principe historique et dogmatique qui préexiste à l'ordre d'apparition des sciences. Dans cette systématisation du savoir les mathématiques, l'astronomie, la physique, la chimie et la biologie représentent un état initial de l'acuité scientifique incarnée par la sociologie qui en constitue la synthèse achevée.

Cet étalement hiérarchique des savoirs résulte, selon Comte, d'une dérive décisive qui aurait fait successivement passer le savoir scientifique d'un stade de généralité décroissante à un état de complexité croissante. L'unité du savoir comme principe circulaire de continuité est donc une figure originelle du travail intellectuel: «Dans l'état primitif des connaissances il n'existe aucune division régulière parmi nos travaux intellectuels, écrit Comte; toutes les sciences sont cultivées simultanément par les mêmes esprits» ${ }^{11}$.

Le développement des connaissances, en modifiant les règles de production du travail intellectuel, a suscité un effritement graduel de cette homogénéité des savoirs. Mais «par une loi dont la nécessité est évidente, chaque branche du système scientifique se sépare insensiblement du tronc, lorsqu'elle a pris assez d'accroissement pour comporter une culture isolée, c'est-à-dire quand elle est parvenue à ce point de pouvoir occuper à elle seule l'activité permanente de quelques intelligences ${ }^{12}$. En somme, la division du travail intellectuel est

11. Auguste Comte, Cours de philosophie positive, T. I, Paris, Au Siège de la Société positiviste, 1982, T.I, p. 22.

12. A. Comte, op. cit. 
devenue un code général de l'activité scientifique qui répartit désormais «entre différents ordres de savants», regroupés selon un fractionnement impératif des connaissances, l'ensemble des «recherches spéciales» ${ }^{13}$. L'esprit de spécialité, conséquemment, est subordonné à l'esprit de discipline qui se projette dans l'aire ordonnée du savoir. Mais la division du travail intellectuel dont Comte reconnaît «les prodigieux résultats» engendre, en revanche, des «inconvénients capitaux (...) par l'excessive particularité des idées qui occupent exclusivement chaque intelligence individuelle» ${ }^{14}$.

En quête d'une sociologie triomphante et d'une science mobilisatrice, Auguste Comte récuse ces divisions «artificielles» entre les disciplines. L'esprit de spécialité auquel la plupart des savants se sont ralliés est mis en cause par Comte qui y voit une tendance «à la considération isolée d'une section plus ou moins étendue d'une science déterminée, sans s'occuper beaucoup de la relation de ces travaux particuliers avec le système général des connaissances positives» ${ }^{15}$.

On voit déjà se profiler ici le projet comtien d'une «solidarité» organique entre les disciplines, unies au plan de l'objet, du statut et de la méthode. Cette solidarité, qui suppose un ordre interne des phénomènes, s'impose à l'ensemble des sciences fondamentales, toutes subordonnées au savoir englobant de la philosophie positive, c'est-àdire de la sociologie ${ }^{16}$. Sous le regard de Comte la dissection engendrée par le déploiement des savoirs spécialisés s'oppose à la réunification du champ des connaissances. La philosophie positiviste s'enferme ainsi dans la poursuite illusoire d'un savoir unitaire dont l'histoire aurait jadis légué la représentation cohérente. Elle s'interdit de la sorte de voir que cet émiettement de la science en une multitude de systèmes indépendants, est la condition à la fois d'un partage social délibéré du savoir, et de son unification matérielle, fixé et symbolisé l'un et l'autre par l'Université. Selon Jean-Paul Resweber.

13. Idem.

14. Ibid., p. 23.

15. Ibid., p. 24.

16. Julien Freund, Les théories des sciences humaines, Paris, PUF, 1973, pp. 69-71. 
"On ne peut, en effet, parler de savoir éclaté qu'en se réclamant d'un lieu utopique d'où l'esprit humain serait censé embrasser la totalité d'un savoir harmonieusement organisé et hiérarchisé. Autrement dit, la division traditionnelle des connaissances est en partie responsable du fait que la spécialisation apparâ̂t inéluctablement comme un morcellement du savoir. Or, une telle division est essentiellement «universitaire» au double sens idéologique et institutionnel. L'Université demeure le symbole qui entretient le rêve d'une possible concentration de la totalité du savoir et qui, en même temps, témoigne de l'irrémédiable fragmentation de ce même savoir en des unités représentant les débris de cette totalisation fictive» ${ }^{17}$.

Le quadrillage unificateur imaginé par la philosophie positiviste et incarné dans la projection épistémologique d'une classification des sciences, constitue dès lors une visée abstraite visant à enrayer «l'influence délétère» de la division du travail intellectuel. Finalement il s'agit pour Comte de dissoudre «l'antique confusion des travaux» dans une réforme de la division du travail, c'est-à-dire dans la formalisation systématique (ou le «perfectionnement») de ses règles. Faisant appel à une idée déjà esquissée dans les Essais sur la philosophie des mathématiques fondamentales, reprise dans l'Opuscule fondamental (1820) et développée plus tard dans les Considérations philosophiques sur les sciences et les savants (1825), Comte conclut qu'il suffit «de faire de l'étude des généralités scientifiques une grande spécialité de plus» ${ }^{18}$.

La synthèse positiviste prend ici tout son sens: mettant fin à la «nécessité provisoire du régime de spécialité scientifique» ${ }^{19}$ comme mode dominant de la division du travail intellectuel et comme principe de sa transformation, elle crée une «classe nouvelle de savants» ${ }^{20}$ délivrée de la vision morcelante héritée historiquement de la philosophie naturelle et désormais en mesure d'interpréter les multiples

17. J.-P. Reswever, op. cit., p. 35.

18. A. Comte, op. cit., p. 24.

19. A. Comte, Cours..., op. cit., T. VI, p. 391.

20. A. Comte, Ibid., T. I, p. 24. 
enchaînements et interférences qui soudent dans une trame commune chacune des sciences positives.

La division du travail ainsi énoncée redéfinit un nouvel horizon épistémologique incompatible avec le conglomérat dispersif du régime des spécialités. D'où ce jugement stigmatisant de Comte qui met face à face l'irrésistible dérive des spécialités et l'expérience synthétique de la méthode positive: «les habitudes dispersives précédemment contractées ont aujourd'hui poussé le régime préliminaire de la spécialité scientifique jusqu'à la plus désastreuse exagération, dogmatiquement justifiée par de vrais sophismes métaphysiques» interdisant «(...) que la philosophie positive soit réellement comprise par personne, puisque chaque section de savants n'en connaît que des fragments isolés dont aucun ne saurait suffire à une conception vraiment décisive» ${ }^{21}$.

La «spécialité dispersive» 22 trouve naturellement son inscription sociale dans la transmission généralisée d'un éthos. Celuici se définit à partir de positivités rationnelles dont la division institutionnelle du travail intellectuel est la représentation sociale. Ainsi, l'emprise sociale des institutions sur l'organisation intellectuelle de l'activité scientifique est-elle à l'origine d'une «anarchie philosophique» exprimée par le «rétrécissement intellectuel» des spécialités, lui-même systématiquement «prolongé par cette stupide résistance académique» dont les «corporations se glorifient si aveuglément» ${ }^{23}$.

Ce qui sous-tend cette dénégation des effets dispersifs des spécialités c'est l'idée d'un magistère spirituel imprégnant l'action du savant. Pour Comte la spécialisation scientifique, purement analytique, ne permet pas d'opérer la synthèse positiviste seule capable d'inspirer les dogmes réformateurs de la société24. En effet, la synthèse comtienne ne répond pas seulement à un souci de vérité épistémologique mais aussi, sinon essentiellement, à un projet de réforme sociale. En d'autres termes, la philosophie positiviste qui

21. A. Comte, Ibid., T. VI, pp. 393-394.

22. Ibid., p. 395.

23. Ibid., pp. 395 et 400 .

24. Voir à ce sujet, R. Aron, Les étapes de la pensée sociologique, Paris, Gallimard, Collection TEL, 1967, pp. 120-121. 
instaure une refonte du système des sciences est subordonnée au système général d'une "politique théorique», de telle sorte que le «spécialiste des généralités» voit son propre rôle transmué dans celui, plus héroïque, du réformateur. Un texte de Comte, datant de 1824, réfere explicitement à cette mutation et indique bien qu'il s'agit «d'une classe spéciale de savants, élevés tout exprès dans la connaissance générale des diverses sciences positives, mais ne cultivant que la science politique» ${ }^{25}$.

On comprend mieux dès lors que le caractère dispersif des spécialités ait dénaturé aux yeux de Comte l'idéal philosophique de la science et, conséquemment, rendu la «classe spéculative» des savants inapte à occuper les fonctions sociales auxquelles elle était naturellement destinée. Le «pouvoir spirituel» du savant est ainsi remis en question, infléchi par la fragilité de son savoir, puisque la condition première de ce pouvoir «consiste assurément en une philosophie pleinement générale, qu'elle qu'en soit la nature» ${ }^{26}$. Le pouvoir spirituel du savant renvoie donc logiquement au pouvoir régulateur de la discipline, c'est-à-dire à sa capacité de structurer à l'intérieur d'une économie générale des spécialités un principe de coordination et de rationalisation.

La discipline se structure autour d'un objet: la concurrence des savoirs. Et si elle impose un ordre au début inconnu mais admis et pourtant reconnu comme légitime, c'est qu'elle présuppose une axiomatique générale à laquelle se seraient dérobées les spécialités. La discipline, estime Comte, ne découpe pas dans la masse uniforme du savoir des zones discursives distinctes correspondant à autant de savoirs spécialisés; au contraire, la discipline s'oppose à la confusion historique des savoirs:

"Toute décomposition quelconque, écrit Comte, devrait nécessairement tendre d̀ déterminer une dispersion correspondante, la répartition fondamentale des travaux humains ne saurait éviter de

25. «Lettre à Valat du 8 septembre 1924», cité par Paul Arbousse-Bastide, in La doctrine de l'éducation universelle dans la philosophie d'Auguste Comte, Tome I, Paris, PUF, 1957, p. 80.

26. A. Comte, op. cit., p. 404. 
susciter, à un degré proportionnel les divergences individuelles et morales, dont l'influence combinée doit exiger, dans la même mesure, une discipline permanente propre d̀ prévenir ou d̀ contenir sans cesse leur essor discordant ${ }^{27}$.

En s'intégrant au régime institutionnalisé des spécialités, la discipline revêt une signification inédite. Dissociée de toute entreprise de compartimentage, la discipline désigne chez Comte, outre un mécanisme de régulation sociale de l'activité scientifique, tout le système des normes doxologiques; celles produites et diffusées à l'intérieur d'un corps scientifique déterminé mais aussi celles qui règlent l'ensemble de l'ordre social réformé. C'est ainsi que:

«Dans toute société vraiment constituée, chaque membre peut et doit être envisagé comme un véritable fonctionnaire public, en tant que son activité particulière concourt à l'économie générale suivant une destination régulière, dont l'utilité est universellement sentie».

La question de la discipline est donc dans la théorie comtienne étroitement liée à celle de la hiérarchie des sciences, dans la mesure où la discipline peut être considérée comme une extension normative de la synthèse positiviste. En assignant à la discipline la fonction de régulariser les efforts dispersifs des spécialités, le modèle positiviste incite les diverses professions savantes, naturellement enclines à ne cultiver que la doctrine isolée d'une seule science, à considérer l'ensemble des vérités particulières propres à chacune des sciences afin d'établir des liens réciproques avec leur spécialité d'appartenance. Ultimement la discipline caractérise une unité compréhensive hiérarchiquement supérieure et qui trouve sa manifestation idéale dans la figure messianique du sociologue. Ainsi, observe Comte dans ses Considérations philosophiques sur les sciences et les savants de 1825:

27. A. Comte, Cours de Philosophie positive, Tome IV, Paris, Édition Reinwald, 1908, p. 317.

28. A. Comte, Ibid., Tome VI, p. 337. 
«Les savants en physique sociale ne feront que s'élever nécessairement, dans la même direction, d̀ un degré immédiatement supérieur à celui des physiologistes. Étudiant une classe de phénomènes qui, par leur nature, dépendent des lois de tous les précédents, ils auront indispensablement besoin d'une éducation préliminaire qui les familiarise avec la connaissance des méthodes et des résultats principaux de toutes les autres sciences positives, seule base rationnelle de leurs travaux propres. Ayant ainsi constamment sous les yeux l'ensemble des connaissances physiques, ils seront inévitablement conduits à construire directement la philosophie positive, aussitôt que leur science spéciale aura fait assez de progrès pour ne plus absorber exclusivement toute leur activité»29.

La discipline telle que conçue par Comte déborde largement la filière épistémologique imposée par le système de classification des sciences et s'inscrit d'emblée dans le projet déjà formulé d'une réforme générale de la société. De telle manière que la discipline ne peut être saisie sous la seule apparence de la distinction épistémologique l'opposant à la spécialité. Procédure de régulation incorporée au projet politique d'une réorganisation sociale, la discipline est ici clairement subordonnée aux finalités du pouvoir. Comte est à cet égard très explicite et le rappelle régulièrement dans le Plan des travaux scientifiques nécessaires pour réorganiser la société de 1822:

«Tout se réduit, en dernier lieu, à faire établir, pour la politique, par les forces combinées des savants européens, une théorie positive distincte de la pratique, et ayant pour objet la conception du nouveau système social correspondant à l'état présent des lumières. Or, en y réfléchissant, on verra que cette conclusion se résume dans cette seule idée: les savants doivent aujourd'hui élever la politique au rang des sciences d'observation ${ }^{30}$.

29. A. Comte, Du pouvoir spirituel, Pierre Aunaud (éd.), Paris, Le livre de poche, 1978, p. 267.

30. A. Comte, Ibid., p. 126. 
D'où, en corrollaire, l'invitation à considérer «la politique comme une science» et à faire admettre, contre la confusion de l'économie politique, que «le véritable rapport direct entre la connaissance de l'organisation humaine et la science politique (...) consiste en ce que la première doit fournir à la seconde son point de départ» ${ }^{31}$.

Bref, si la doctrine positiviste de Comte n'aboutit pas à la reconnaissance d'une intelligibilité épistémologique de la notion de discipline, elle souligne en contrepartie la nature politique de tout système porteur d'un ordre intrinsèque ou prétendu tel, fusse celui de la discipline.

\section{Sociologie de la discipline}

La discipline, on l'a dit, est un protocole de contrainte. Mais ce qu'elle impose, à partir du XIXe siècle en particulier, c'est moins un certain ordre conceptuel qu'une orthodoxie sociale érigée autour d'un corpus rationnel. Autrement dit, la discipline ne se structure plus seulement sur la base d'un ensemble complexe de pratiques discursives mais autour d'un enjeu social, la monopolisation de l'autorité scientifique. C'est dire à quel point la notion de discipline paraît prosaïque aussitôt qu'on tente de la définir à partir d'un objet conceptuel pré-existant. En réalité, la discipline est un savoir constamment redéfini et profondément lié à la logique du système de reproduction des connaissances.

Parce qu'elle est d'abord un artefact du système de la science, la notion de discipline ne permet pas de «désenchanter» du monde, selon l'expression de Weber. Elle est devenue une figure insolite au moment même où elle cessait de délimiter à l'avance des espaces topologiques exclusifs. Ainsi dépouillée de toute identité épistémologique définitive, la discipline se voit progressivement pourvue d'une identité sociale que nul raisonnement ne parvient à élucider totalement. Cette ambivalence structurale de la discipline est évoquée par J.D. De Certaines lorsqu'il

31. Ibid., pp. 129 et 199. 
écrit: «sociologiquement, la biophysique existe (...). Pourtant, nul n'est capable de la définir, de préciser sa situation épistémologique» ${ }^{32}$.

Aussi bien la discipline ne peut plus se définir à partir d'un objet qu'elle s'approprierait comme un domaine naturel, en vertu d'un découpage des objets scientifiques qui épuiserait ultimement la réalité. Cette délimitation par la discipline d'un «objet central», Pierre Favre n'hésite pas à dire, en se fondant sur le cas de la science politique, qu'elle «n'a pas épistémologiquement de sens» ${ }^{33}$. Ce qui est vrai pour la science politique l'est également pour l'ensemble des disciplines anthropologiques qui ne peuvent elles non plus prétendre à aucune forme de monopole épistémologique dans leurs sphères respectives de réflexion. C'est d'ailleurs les dimensions philosophico-sociales de ce débat que soulève $\mathrm{C}$. Castoriadis lorsqu'il écrit au sujet des disciplines anthropologiques:

«Ici les effets de la séparation des disciplines se font sentir plus lourdement que partout ailleurs: de la séparation de la philosophie (qui, à vrai dire, n'a jamais pu s'accomplir effectivement), puisqu'elle conduit a oublier les innombrables présupposés et implications philosophiques de tout discours anthropologique; de la séparation des autres grands ensembles de disciplines, physiques et surtout biologiques, puisqu'il est impossible de voir dans la nature physique et biologique de l'homme une simple condition abstraite de son entité historique; de la séparation entre disciplines anthropologiques, enfin, puisqu'ici l'unité de l'objet défie immédiatement la dissection scientifique et que l'on peut se demander si la distinction que nous

32. J.D. De Certaines, «La biophysique en France: critique de la notion de discipline scientifique», in Perspectives on the Emergence of Scientific Disciplines, G. Lemaine, R. MacLeod, M. Mulkay, P. Weingart (éds.), Mouton-The Hague, Paris-Aldine, Chicago, 1976, p. 99.

33. P. Favre, «La question de l'objet de la science politique a-telle un sens?», in Mélanges dédiés à Robert Perroux, Lyon, Editions L'Hermès, 1980, p. 128. 
faisons entre disciplines différentes a un sens pour des sociétés autres que la nôtre» ${ }^{34}$.

S'il est vrai que la notion de discipline scientifique n'acquiert sa véritable portée socio-épistémologique qu'au moment de l'émergence des sciences humaines, en revanche l'idéalisme des articulations disciplinaires n'appartient pas en propre aux sciences humaines même si ses effets y semblent plus spontanément visibles que dans les sciences de la nature. Car, en effet, la physique et la biologie sont elles aussi réfractaires au découpage abstrait de la discipline qui tient pour permanent et immuable un état de développement provisoire et inachevé. C'est cette représentation d'une discipline existant en soi et tout entière absorbée dans la masse transcendante d'une philosophia perennis que Jean-Marc Lévy-Leblond met en accusation lorsqu'il constate que:

«La physique (...) comme tout autre science d'ailleurs, ne peut être définie une fois pour toutes, de façon abstraite et définitive, par référence, par exemple, à sa «méthode», et moins encore aux «objets» de son étude: au contraire, c'est précisément le travail scientifique qui, à partir du réel étudié, construit ses objets propres. La meilleure preuve de ces effets idéologiques est fournie par l'évolution historique de chaque discipline, ses modifications de frontières, éventuellement de nomi ${ }^{35}$.

Le modèle de la discipline développé au cours du XIXe siècle ne résiste pas à l'explication historique. Ainsi en est-il, par exemple, de l'opposition historique instaurée entre les sciences et les lettres qui

34. C. Castoriadis, Les carrefours du labyrinthe, Paris, Seuil, 1978, p. 186.

35. J.M. Lévy-Leblond, "Mais ta physique?», in L'idéologie deldans la science, H. Rose et al. (éds.), Paris, Seuil, 1977, p. 245. Cette conception de la discipline n'est pas nouvelle puisqu'elle fait historiquement écho, parmi d'autres sources, à l'axiome constructiviste de Bachelard qui affirmait: "Rien ne va de soi. Rien n'est donné. Tout est construit." (La formation de l'esprit scientifique, Paris, Librairie philosophique, J. Vrin, 1977, p. 14.). 
n'est plus, d'où qu'on l'observe, qu'une survivance idéologique. L'origine de cette dissociation trouve apparemment son origine dans la distinction scolastique entre les arts du trivium, réunissant la grammaire, la rhétorique et la dialectique, et les arts du quadrivium, formés de l'arthmétique, la musique, la géométrie et l'astronomie ${ }^{36}$. Mais l'arrivée des virtuosi britanniques, consacrée en 1662 par la création de la Société Royale de Londres, vient rompre la division philologique qui oppose encore à ce moment le clerc médiéval et le lettré romanisant. Aussitôt «la science nouvelle ne reconnaît d'autre autorité que celle de la méthode rigoureuse et des faits dûment constatés» ${ }^{37}$, déniant ainsi toute valeur rationnelle aux divisions dogmatiques consacrées par l'humanisme traditionnel.

La division sociale du travail parmi les membres de la Société Royale n'empruntera donc jamais, suivant l'analyse de R.K. Merton, le modèle de la disjonction scolastique; en conséquence l'activité scientifique, désormais déliée de toute nécessité académique pourra s'orienter indifféremment vers des secteurs appartenant aux sciences

36. G. Gusdorf, De l'histoire des sciences à l'histoire de la pensée, Paris, Payot, 1966, pp. 19 et suiv. La dualité opposant les lettres et les sciences apparaît aujourd'hui comme un véritable contresens épistémologique puisque, peu à peu, les unes et les autres tendent à devenir, par l'utilisation d'un système complexe d'énonciation, des décalques mutuels. Selon la formule d'Abel Guggenheim, «la production scientifique est simplement une forme littéraire particulière» («Quand les sociologues étudient la littérature scientifique», La Recherche, vol. 13, $\mathrm{n}^{\circ} 137$, octobre 1982, p. 1200). Cette idée a été notamment illustrée par B. Latour et $S$. Woolgar dans le livre Laboratory Life, the Social Construction of Scientific Facts, (Beverley Hill, Sage, 1979), où la science est décrite comme un système organisé d'écriture obéissant à un code spécifique de composition. Sur les formes de compétence linguistique telles qu'elles s'exercent dans les articles scientifiques, voir l'analyse de B. Latour et P. Fabbri, «La réthorique de la science», Actes de la recherche en sciences sociales, $\mathrm{n}^{\circ} 13$, février 1977, pp. 81-95. Sur le thème plus général de la scientificité de la critique dont est investie le texte littéraire, voir l'article de Martine Léonard, «De la science comme cliché, ou comment penser la littérature», Études françaises, vol. 19, n 21 , automne 1983, pp. 97 110.

37. G. Gusdorf, op. cit., p. 26. 
«pures» ou, au contraire, vers les sciences d'application ${ }^{38}$. À l'exception de la France qui crée deux académies distinctes de science et de littérature, les autres pays européens imitent le modèle britannique qui regroupe sous l'appellation générique de «Science» l'ensemble du champ des connaissances. Ainsi, dès 1700, l'Électeur Frédéric de Prusse fonde la Société Royale des Sciences alors que la Société Royale des Sciences de Goettingen est créée en 1751 dans le royaume du Hanovre.

En même temps qu'elle favorise l'émergence d'une activité scientifique progressivement libérée de l'amateurisme et du «thêâtre» scientifique $^{39}$, la création des sociétés savantes européennes constitue l'amorce d'une longue réflexion pédagogique sur l'enseignement scientifique. Cette réflexion aboutira, plus tard, à la prééminence des disciplines scientifiques plutôt qu'à la consécration de l'unité pédagogique des disciplines. En France, par exemple, la réforme napoléonienne de l'Université sanctionne juridiquement la séparation entre la faculté des lettres et les facultés des sciences. L'Université impériale - et avec elle l'École polytechnique, créée par Napoléon pour répondre aux pressions politiques d'un Empire dont les visées expansionnistes exigeaient qu'on lui donnât sans cesse son cortège d'ingénieurs militaires, d'administrateurs et d'officiers d'artillerie -

38. R.K. Merton, Science, Technology and Society in 17th Century, England, N.Y., Fertig, 1970.

39. La science du XVIIIle siècle, d'une manière générale «n'est pas une vie, pas même un métier» selon la déclaration de Bachelard qui n'est pas sans évoquer les remarques formulées un siècle plus tôt par le mathématicien anglais Charles Babbage, lequel déplorait que la pratique de la science dans l'Angleterre du XIXe siècle ne fût pas considérée par les élites politiques comme une «profession distincte» au même titre que les professions libérales (Reflections on the Decline of Science in England, and on Some of Its Causes, Londres B. Fellowes, 1830). L'exemple du «dîner électrique» de Franklin en 1748, tel que raconté par J. Priestley dans The History and Present State of Electricity ou celui du laboratoire de madame La Marquise du Châtelet au Château de Cirey, illustre selon Bachelard l'état réel de la science du siècle de l'Aufklarung, laquelle se débat encore dans un vaste "théâtre» où la vérité se définit dans les «minauderies» de la représentation (Bachelard, op. cit., pp. 3334). 
manifeste l'ampleur de la réflexion pédagogique qui traverse l'ensemble des pays européens. Celle-ci renforce le prestige social de la science au moment même où s'intensifie le déclin de l'enseignement classique.

Désormais «une séparation stricte des sciences donne à leur spécialisation un caractère de rigueur et un gage d'efficacité» ${ }^{40}$ qu'un nouveau modèle éducatif vient sanctionner. La spécialisation du savoir coïncide donc avec la révolution pédagogique de la fin du XIXe siècle à laquelle répond la formule de Durkheim, prononcée en 1888, affirmant qu'«une science n'est vraiment constituée que quand elle est divisée et subdivisée» ${ }^{41}$.

D'un point de vue strictement épistémologique on peut penser, comme Jean Piaget, que la différenciation théorique conventionnellement admise entre les sciences naturelles et les sciences de l'homme (incluant les lettres) donne lieu à «toute une somme d'approximations successives» qui rendent peu pertinente une analyse fondée sur une «simple dichotomie» des systèmes. Ainsi, les propriétés du «sujet» font-elles apparaître la grossièreté théorique de cette ancienne antinomie. Par exemple, la biologie définit simultanément le «sujet» en termes de «variations aléatoires ou mutation» et de «sélection imposée par le milieu», établissant de cette façon une «soudure» épistémologique entre les Naturwissenschafter (sciences de la nature) et les Geisteswissenschaften (sciences humaines). Par ailleurs, les sciences de la nature et les sciences humaines sont associées dans un processus d'échange des méthodes et de transitivité des techniques (e.g. le passage des structures logicomathématiques des sciences naturelles aux sciences humaines ou l'emprunt de la théorie de l'information à la thermodynamique) qui a tendance à «naturaliser» et à «humaniser» certains processus naturels. Enfin, la classification linéaire des sciences ignore les connexions logiques qui se sont tissées entre les différentes sciences: «en réalité, écrit Piaget, aucune des sciences ne peut être étalée sur un plan unique

40. S. Moscovici, Essai sur l'histoire humaine de la nature, Paris, Flammarion, coll. Champs, 1977, p. 433.

41. Cité par P. Favre, "L'absence de la sociologie politique dans les classifications durkheimiennes des sciences sociales», Revue française de science politique, vol. XXXII, $\mathrm{n}^{\circ} \mathrm{I}$, février 1982, p. 7. 
et chacune d'entre elles comporte des niveaux hiérarchiques» quant à son objet, ses interprétations conceptuelles, son épistémologie interne ou dérivée ${ }^{42}$. De telle sorte que la division formelle tracée entre les sciences, en particulier entre les sciences et les lettres, ne trouve aucune justification solide dans l'explication épistémologique. Du point de vue de l'explication sociologique, la mise en cause de l'existence de ce clivage repose sur des justifications contradictoires. Du côté des sciences de la nature, la dénégation du clivage passe par l'affirmation de l'unité de la science qui n'a elle-même de sens qu'en référence à l'unité du réel. Du côté des sciences de l'homme, cette même dénégation a d'autres enjeux, notamment celui de légitimer leur propre revendication de scientificité.

\section{De la discipline, de la spécialité}

La spécialisation marque donc l'éclatement de la discipline conglomérat, c'est-à-dire d'un corps de savoir unifié et doté de structures cognitives immanentes. En réalité, la discipline ne résiste pas aux effets dispersifs de la division sociale du travail scientifique. La discipline s'est ainsi transformée en un sous-produit d'une science qui a renoncé à son unité. Durkheim, prophétique, ne s'y était pas trompé: la spécialisation a fait pénétrer le savant «non seulement dans une science particulière mais dans un ordre spécial de problèmes (...) la science, morcelée en une multitude d'études de détails qui ne se rejoignent pas, ne forme plus un tout solidaire ${ }^{43}$.

La spécialité est devenue un maître-mot de la sociologie sans pour autant dissiper l'ambiguité née à la suite de la formation du

42. J. Piaget, Épistémologie des sciences de l'homme, Paris, UNESCO-Gallimard, Coll. Idées, 1970, pp. 91-104.

43. E. Durkheim, De la division du travail social, Paris, PUF, 1967 , p. 347. Dans un ouvrage paru après sa mort, Durkheim aborde à nouveau ce thème: "Chacun, écrit-il, s'enferme dans sa spécialité, professe la science de son choix comme si elle était la seule, comme si elle était une fin, alors qu'elle n'est qu'un moyen en vue d'une fin à laquelle elle devrait être à tout moment subordonnée» (L'évolution pédagogique en France, T. II, Paris, Alcan, 1938, p. 13). 
couple discipline/spécialité. Trop souvent, en effet, la spécialité a été définie comme l'envers parfaitement symétrique de la discipline, confondant ainsi différents modes d'institutionnalisation de l'activité scientifique. Quelques remarques s'imposent encore à ce propos.

Richard Whitley, dans un article célèbre, a tenté d'expliquer à partir de deux concepts majeurs (ceux de disciplines parapluies et de disciplines polythéistiques) la nature de l'écart séparant les deux phénomènes. Selon Whitley, la discipline «est le mode principal d'institutionnalisation sociétale de la recherche en science» ${ }^{44}$. À cet égard la discipline coordonne un ensemble unifié de valeurs autour d'un aspect particulier de la réalité; elle instaure un «principe d'ordre», c'està-dire l'espace cognitif à l'intérieur duquel une spécialité se développe et conçoit ses modèles explicatifs. La discipline confêre ainsi à une unité de recherche particulière un ordre général d'attitudes face à la science, bref les éléments fondamentaux d'un éthos institutionnalisé.

La spécialité, en revanche, est orientée vers la définition de modèles explicatifs, conséquemment les aires de recherches et les spécialités sont faiblement institutionnalisées et les interactions entre elles sont peu fréquentes. En somme, la spécialité ne recouvre pas une communauté solidaire édifiée sur la base de principes d'engagement («commitment») cognitifs structurés.

Les disciplines parapluies confèrent peu d'identité disciplinaire aux spécialistes qu'elles regroupent puisque dans leur cas le travail scientifique s'organise principalement sur des aspects divergents d'une discipline. Dans ce cas la production de la recherche se développe prioritairement autour de la spécialité et de ses aires de recherche affiliées sans association directe à une discipline maîtresse qui l'orienterait. La spécialité se définit ici comme la source prédominante de l'identité intellectuelle et sociale.

Dans le cas des disciplines polythéistiques, les bases discursives de la discipline sont liées plus directement aux débats courants de la spécialité. Conséquemment, la recherche est ici moins autonome puisque les implications cognitives et les engagements métaphysiques,

44. R. Whitley, «Umbrella and Polytheistic Scientific Disciplines and their Elites», Social Studies of sciences, 6 (1976), p. 475. 
à tous les niveaux des aires de recherches, sont fortement liés à la structure disciplinaire. Ainsi la conduite d'une recherche aboutit à la formation d'une identité disciplinaire, ou de ce que nous serions tentés de qualifier d'habitus disciplinaire.

Dans la pensée de Whitley la discipline apparaît donc comme un principe organisateur régissant les aires de la recherche institutionnalisée. Le sociologue Daryl E. Chubin, dans une revue critique de la littérature sociologique consacrée à la notion de spécialité $^{45}$, distingue lui aussi la discipline - désignée comme le domaine de l'enseignement de la science - de la spécialité - décrite comme l'unité minimale du domaine de la recherche, ce que Warren $\mathbf{O}$. Hastrom sumomme le «micro-environnement de la recherche» ${ }^{46}$.

C'est donc sur la base de réseaux sociaux restreints, peu ou fortement institutionnalisés, que se fonde la distinction opposant la notion de discipline à celle de spécialité. La spécialité désigne en l'occurrence un processus social de fragmentation - ou de différenciation - de la discipline: «La différenciation est ainsi perçue», selon Ron Johnston et Dave Robbins, «comme un pur processus social dérivé de la compétition à l'intérieur de la communauté» ${ }^{47}$ scientifique. Pourtant la spécialisation s'impose, avec la fragmentation, comme l'un des deux processus dominants de différenciation de l'activité scientifique. Dans cette perspective, la spécialisation apparaît selon les auteurs comme l'un des aspects prioritaires de la division du travail, déterminée simultanément par l'opérationnalisation des sources de pouvoir et par l'autorité interne de

45. D.E. Chubin, «The Conceptualization of Scientific Specialties», The Sociological Quarterly, 17, automne 1976, pp. 448476.

46. W.O. Hagstrom, «Factors Related to the Use of Different Modes of Publishing Research in Four Scientific Fields», in Carnot E. Nelson et D.K. Pollock (éds.), Communication Among Scientists and Engineers, Lexington, Mass., Lexington Books, pp. 85-124, cité par D.E. Chubin, ibid., p. 450.

47. R. Johnston, D. Robbins, «The Development of Specialties in Industrialised Science», The Sociological Review, vol. 25, 1977, p. 89. 
la communauté scientifique ${ }^{48}$. C'est pourquoi la spécialisation reste profondément liée aux mécanismes internes de la science ainsi qu'au contrôle collégial qu'elle exerce. À l'opposé de ce processus d'autonomisation de la science, la fragmentation s'affirme comme un mécanisme de division du travail subordonné à des facteurs sociaux externes, tel le contrôle patronal. Cette forme particulière de différenciation, dépendante de groupes occupationnels fragmentés et hiérarchisés, met directement en cause la cohésion sociale de la communauté scientifique. Elle tend en effet à dissoudre dans la détermination d'intérêts locaux l'intelligibilité cognitive unissant les divers champs de la science.

En somme, alors que le contrôle collégial interne conduit à l'élaboration d'un modèle de différenciation qui intègre l'activité professionnelle des groupes spécialisés sur la base du principe durkheimien de la solidarité organique, le contrôle patronal externe soumet pour sa part l'activité scientifique à un processus de différenciation qui entraîne une prolifération de sous-groupes fortement spécialisés et irréductibles à toute tentative d'intégration fonctionnelle.

Le texte de Johnston et Robbins met ainsi en lumière l'un des aspects majeurs de l'activité scientifique moderne: le contrôle collégial des activités occupationnelles en science s'exerce dans le champ traditionnel de la compétition sociale et de ses valeurs régulatrices. La discipline ne peut plus, dans ces conditions, se définir comme une entité cognitive abstraite qui déterminerait des totalités distinctes dans le champ des connaissances. Loin d'apparaître comme un corpus autonome, la discipline se révèle de plus en plus comme un habitus disciplinaire ${ }^{49}$, c'est-à-dire comme un système de dispositions

48. Ibid., pp. 97-99.

49. P. Bourdieu définit ainsi dans Le sens pratique, Paris, Éditions de Minuit, 1980 , p. 80 , l'ensemble des conditionnements produits par l'habitus: «systèmes de dispositions durables et transposables, structures structurées prédisposées à fonctionner comme structures structurantes, c'est-à-dire en tant que principes générateurs et organisateurs de pratiques et de représentations qui peuvent être objectivement adaptées à leur but sans supposer la visée consciente de fins et la maîtrise expresse des opérations nécessaires pour les atteindre 
objectives et inconscientes qui, à l'intérieur d'un espace cognitif institutionnellement déterminé et prescrit (l'enceinte de la faculté, du département, du laboratoire, etc.) oriente la pratique et le mode de représentation intellectuelle des agents au sein d'un groupe dont les activités sont instantanément ajustées aux normes doxologiques d'un champ scientifique déterminé $e^{50}$.

L'habitus disciplinaire universalise dans la conscience privée d'un acteur un code général conforme au paradigme dominant d'une communauté particulière. La fragmentation (ou la subdivision) de la discipline en une multitude de sous-spécialités regroupées autour d'une identité professionnelle distincte, a façonné les bases d'un habitus disciplinaire dans le champ scientifique. L'inculcation de l'habitus disciplinaire correspond, dans l'expression isntitutionnalisée de la discipline, à ce système «d'enveloppement continu» ${ }^{51}$ décrit par Durkheim et dont le modèle allemand nous fournit un exemple significatif.

Le modèle allemand du début du siècle, dont le système éducatif reposait sur l'organisation de laboratoires et d'instituts spécialisés affranchis des corporations médicales et des écoles d'ingénieurs, a profondément inspiré tout l'Europe. Il peut, à cet égard, être considéré comme l'archétype d'une nouvelle pédagogie scientifique qui précipitait ses adeptes dans un univers social et intellectuel saturé de règles et de conduites prétablies.

À l'horizon de l'ère whilhelmienne (1890-1918), comme l'explique Lewis Pyenson, les scientifiques allemands furent soumis à un mécanisme institutionnel qui agissait comme un code restrictif et engendrait des conduites communes. En conséquence:

(...)». Voir également du même auteur, «Champ du pouvoir, champ intellectuel et habitus de classe», Scolies, 1, 1971, pp. 7-26.

50. Rappelons que dans la théorie du champ scientifique telle qu'élaborée par Pierre Bourdieu, la discipline n'est que la projection à l'échelle réduite d'un champ scientifique plus vaste: «Il y a, écrit-il, à chaque moment, une hiérarchie sociale des champs scientifiques - les disciplines - (...)» «La spécificité du champ scientifique et les conditions sociales du progrès de la raison», Sociologie et sociétés, vol. $7, \mathrm{n}^{\circ} 1$, mai 1975 , p. 96 .

51. E. Durkheim, L'évolution... op. cit., p. 108. 
«Les physiciens et les astronomes connus publiaient des articles techniques dans des revues spécialisées et prononçaient des discours ex cathedra devant des centaines de disciples, pour qui l'avancement, y compris les promotions matérielles, dépendait en grande partie du comportement. Un disciple devait faire la preuve d'une pensée correcte lorsqu'il abordait les problèmes fondamentaux débattus dans les revues spécialisées. Sa nomination dépendant le plus souvent d'une décision d'État, il devait également proclamer son adhésion a l'orthodoxie dans les domaines aussi bien religieux que politiques et sociaux. En Allemagne whilhelmienne les disciplines scientifiques conditionnaient la vie entière de ceux qui s'y consacraient. Il s'agissait d'un apprentissage à l'issue duquel le scientifique était prêt à assumer le modèle de pensée de sa discipline. (...) Ce système, celui des disciplines scientifiques, permit une activité orientée et suivie, enracinée dans un style de vie socialement accepté» 52 .

À la lumière de cet exemple l'habitus disciplinaire peut être aussi compris comme un mécanisme par lequel la culture scientifique institutionnalisée régit la pensée et la conduite des scientifiques. Toutefois, cette opération ne se déroule pas, selon le modèle proposé par T. Kuhn, sous l'horizon d'un paradigme qui fournirait un modèle unique (de valeurs et de solutions des énigmes) à l'activité scientifique d'un groupe déterminé. Malgré cette réserve, la conception kuhnienne du paradigme n'est pas totalement dénuée d'utilité dans la mesure où

52. L. Pyenson, «La place des sciences exactes en Allemangne à l'époque de Guillaume II», Europa: Revue d'études interdisciplinaires, vol. 4, 1981, p. 188. Dans son Esquisse d'une psychologie des classes sociales (Paris, Librairie Marcel Rivière et Cie, 1964), Maurice Halbwachs définit très précisément les éléments d'un habitus (ou d'un éthos) soumis au procès de production et de reproduction des connaissances. Les savants, écrit-il, «sont sortis des collèges, ils ont travaillé dans les laboratoires, vécu dans des milieux scientifiques, profité de tout un ensemble d'institutions qui ont pour objet de former et de maintenir, distincte de toutes les autres et se suffisent avec son langage, ses conventions, ses traditions aussi, ce qu'on pourrait appeler la société des savants» (p. 230). 
celui-ci sert de support à la structure du groupe, ou mieux encore, dans la mesure où il incarne le groupe. «Un paradigme, écrit Kuhn, est ce que les membres d'un groupe scientifique possèdent en commun, et, réciproquement, un groupe scientifique qui se compose d'hommes qui se réferent au même paradigme» ${ }^{53}$.

Cependant, la surdétermination du paradigme tel que défini par Kuhn laisse dans l'ombre les conditions d'émergence du groupe et exclut de son analyse les groupes formés sans référence immédiate à un paradigme dominant. En ce sens, le paradigme apparaît comme une extension philosophique de la notion de discipline, puisqu'il implique la constitution et la reconnaissance préalable d'un objet commun d'une théorie ou d'une loi - appartenant à la «science normale». Or, nous l'avons vu plus tôt, depuis le XIXe siècle, la discipline ne se forme plus autour d'un noyau discursif central (conséquemment, la permutabilité des frontières épistémologiques peut être envisagée dans le cadre de la science normale et non plus uniquement dans le brouhaha prophétique d'une révolution scientifique) mais s'édifie, au contraire, dans la représentation matérielle du groupe. De fait, l'inscription sociale véritable de la discipline c'est le groupe en tant que microinstitution.

Paradoxalement, Kuhn reconnaît que «les principales disciplines sont des groupes» ${ }^{54}$ dont les limites peuvent être retracées à partir des attributs sociaux de leurs membres. Afin de résoudre l'ambiguiité suscitée par la notion de paradigme Kuhn lui a substitué, dans sa Postface de 1969, la notion de «matrice disciplinaire», laquelle suppose l'adhésion commune de spécialistes à une discipline particulière et la possession d'un certain nombre d'éléments ordonnés (les généralisations symboliques, les paradigmes métaphysiques, les valeurs) constituant le paradigme de la discipline ${ }^{55}$. À l'opposé du paradigme qui demeure un concept autonome, préexistant au groupe, la matrice disciplinaire introduit l'idée d'une relation continue entre un corpus doctrinal spécifique et l'activité occupationnelle d'un groupe.

53. T. Kuhn, La structure des révolutions scientifiques, Paris, Flammarion, 1972, p. 208.

54. Ibid., p. 210.

55. Ibid., pp. 215-218. 
Pourtant, même reformulée, le concept de paradigme ne fournit pas un modèle heuristique entièrement satisfaisant. La prolifération des sousgroupes spécialisés au sein des disciplines scientifiques rend désuète en effet la description idéalisée d'une communauté scientifique consensuellement regroupée autour d'un paradigme ou d'une série de paradigmes. Cette espèce de convergence paradigmatique, on l'a constaté, n'est qu'une abstraction théorique. En réalité, les groupes se forment non pas sur la base d'une discipline, mais autour d'un ensemble de micro-paradigmes qui, d'un groupe à l'autre, au sein d'une même discipline, sont dans un rapport d'exclusion réciproque ${ }^{56}$. De telle sorte que la discipline est aujourd'hui un objet décentré où toute l'activité se trouve projetée à la périphérie, c'est-à-dire aux confins éclatés des spécialités.

Par ailleurs, le concept de matrice disciplinaire reste fortement descriptif puisqu'il ne permet pas d'analyser le processus de disciplinarisation des groupes constitués. Ce processus met en cause non pas un espace épistémologique déterminée - même si la constitution du groupe suppose implicitement la reconnaissance commune d'un tel espace - mais bien davantage la formation d'un groupe capable d'imposer légitimement sa définition des enjeux scientifiques. L'habitus disciplinaire rend compte, pour sa part, du rôle des mécanismes de socialisation en science car il est inséparable des modes de «transmission institutionnalisée de la culture» mis au jour par P. Bourdieu. À ce titre, il tente de prendre pour objet «les individus

56. La spécialisation des savoirs et leur propension à se constituer à partir de micro-concepts qui les isolent les uns des autres est telle, en effet, qu'elle paraît mettre en jeu non seulement l'existence des regroupements scientifiques pluridisciplinaires, mais aussi celle des sociétés savantes unidisciplinaires. Soulignons, à titre d'exemple, que dès 1943, Léo Pariseau, alors président de l'ACFAS, prédisait et appelait la disparition de ce type d'association. Empruntant les mots d'un confrère français il déclarait: «Mais considérons que les chimistes ont depuis longtemps leurs congrès particuliers où les «minéraux» n'écoutent que d'une oreille distraite les communications des «organiciens» et vice versa. Il en va de même de toutes les autres «spécialités» du savant». («Et avant l'ACFAS, il y eut la SPASLAC», Les Annales de l'ACFAS, vol. 9, 1943, pp. 193-194). 
«programmés», c'est-à-dire dotés d'un programme homogène de perception, de pensée, d'action (...) les hommes formés à une certaine discipline ou à une certaine école (...) façonnés selon le même «modèle» (pattern) $)^{57}$.

L'habitus disciplinaire ne se déploie pas dans un environnement sans rupture et sans constestation, il appartient à l'univers hétérogène de la discipline. En conséquence, l'habitus disciplinaire se trouve ouvert à une série d'enjeux institutionnels. Dans un autre article, Alberto Cambrosio et Peter Keating développent à partir du cas de la chronobiologie l'idée d'une «forme disciplinaire», désignée comme un «enjeu disciplinaire» pour «la reconnaissance de la légitimité de l'exercice du contrôle professionnel». La distribution différentielle de légitimité mise en forme par l'enjeu disciplinaire repose sur la dynamique interne de la forme disciplinaire, puisque cet enjeu est caractérisé «par le pouvoir, supporté par les producteurs, de définir le corpus doctrinaire à être transmis, le curriculum, les règles d'apprentissage et finalement les méthodes de certification et de sanction ${ }^{58}$.

La notion de forme disciplinaire rompt avec le modèle taxonomique de la discipline et tente de ressaisir les contenus éphémères de la discipline. La forme disciplinaire est une configuration sans cesse contestée et redessinée par les enjeux disciplinaires qui animent le champ scientifique. Dans ces conditions un corpus doctrinal déterminé (la physique, la chimie, la sociologie) ne peut s'attribuer le statut de discipline - et celui-ci sera nécessairement provisoire qu'en vertu de l'état des luttes au sein du champ. En effet, «les disciplines, précisent les auteurs, peuvent être conçues comme une forme de lutte entre les autres disciplines dans le champ», c'est-à-dire comme une lutte institutionnelle ayant pour enjeu spécifique la

57. P. Bourdieu, «Systèmes d'enseignement et systèmes de pensée», Revue internationale des sciences sociales, vol. $\mathrm{XIX}, \mathrm{n}^{\circ} 3$, 1967 , p. 369.

58. A. Cambrosio, P. Keating, «The Disciplinary Stake: the Case of Chronobiology, Social Studies of Science, vol. 14, 1983, p. 328. 
capacité «d'exercer un contrôle «collégial» de la production et de la reproduction des connaissances» ${ }^{59}$.

La notion d'enjeu disciplinaire, qui permet ainsi de mieux saisir le sens exact de l'habitus disciplinaire, suppose bien sûr l'intériorisation tacite d'un ensemble de déterminations antérieures juxtaposées (lieu de formation, esprit de corps, position hiérarchique de la discipline, mode de pensée, etc.). Cependant, l'habitus disciplinaire n'est pas identifié par un paradigme statique dont la cohérence interne ne serait jamais sujette à la dénégation. Au contraire, cet habitus est soumis à l'action déformante d'enjeux institutionnels multiples qui sont susceptibles à tout instant de modifier la nature de ses projets, puisque c'est là l'une de ses caractéristiques les plus profondes: l'habitus disciplinaire est le produit d'une série d'actes passés, mais il ne poursuit pas de fins apodictiques dictées par un passé qui l'engloberait. Aussi peut-on le qualifier, suivant une terminologie employée par Raymond Boudon, comme un paradigme de type interactionniste, par opposition à un paradigme de type déterministe où les «actes sont exclusivement expliqués par des éléments antérieurs à ces actes» ${ }^{60}$.

L'habitus disciplinaire, à l'inverse, n'est pas le résultat exclusif d'un certain nombre de dispostions antérieures, pas plus par ailleurs qu'il n'est intégralement réductible à leur description. L'état d'un habitus disciplinaire, à un moment particulier de son histoire, n'est pas la seule conséquence d'événements antérieurs, mais aussi la manifestation concrète d'enjeux opposés et toujours redéfinis à l'intérieur du champ scientifique. Bref, l'habitus disciplinaire n'est pas assignable à des fins choisies ou prévues, d'où l'existence de luttes, de conflits, de déviances et de comportements hétérodoxes dans le champ où il se manifeste.

59. Ibid., p. 347.

60. R. Boudon, Effets pervers et ordre social, Paris, PUF, 1977, pp. 189-252. L'auteur, d'autre part, définit en ces termes le paradigme: «Le langage dans lequel sont formulés les théories ou éventuellement des sous-ensembles importants de théories émises dans le cadre d'une discipline» (p. 190). 


\section{Conclusion}

Nous avons voulu montrer dans cet article que la discipline scientifique n'est pas cette complexité intrinsèque que certains continuent d'imaginer naturellement enchâssée dans la science. La notion d'habitus disciplinaire veut précisément rompre avec ce dualisme stérile qui met en opposition une conception internaliste de la discipline, qui isole la connaissance de son contexte d'élaboration, et une conception externaliste qui s'emploie à l'inverse à la réduire à ses dimensions sociales ou historiques. La notion d'habitus disciplinaire renvoie dos à dos ces visions déterministes de la discipline. Elle montre que la connaissance (ou les contenus de la science) s'élabore par une suite de constructions opératoires et de liens sociaux constamment renégociés.

Un récent courant ethnolosociologique ${ }^{61}$ a montré que ces liens sociaux s'échafaudent moins autour d'un noyau dur de l'activité scientifique qu'à partir de réseaux complexes de compétences, d'intérêts ou de croyances participant à la production des connaissances.

Dans ce contexte, la discipline apparaît régie par le jeux de contingences extérieures dont l'influence reste toutefois «limitée» et «négociable», ainsi que par l'action de contraintes imparfaites et réciproques s'exerçant entre le noyau dur et les acteurs ${ }^{62}$. L'habitus disciplinaire dévoile ainsi une discipline qui est le résultat d'une lente construction socio-technique et dont l'ensemble des connaissances produites est «la conséquence de la mobilisation de réseaux hétérogènes» 63 .

61. Voir en particulier: K. Knorr, The Manufacture of Knowledge, Oxford, Pergamon, 1981; M. Lynch, Art and Artifact in Laboratory Science, Londres, Routledge and Kegan Paul, 1985; B. Latour et Woolgar, La vie de laboratoire, Paris, Éditions la Découverte, 1988; B. Latour, Science in Action, Milton Keynes, Open University Press, 1987.

62. M. Callon et J. Law, «La protohistoire d'un laboratoire», in M. Callon (éd.), La science et ses réseaux, Paris, Éditions la Découverte/Conseil de l'Europe/UNESCO, 1989, pp. 66-67.

63. M. Callon et J. Law, op. cit., p. 114. 
La construction de la discipline implique donc non seulement la reconnaissance d'un objet distinct mais aussi le pouvoir d'en imposer les conditions sociales d'élaboration et d'apprentissage.

C'est ainsi que la notion d'habitus disciplinaire nous révèle l'existence d'une filière continue de l'un à l'autre. Dans cette perspective, la discipline resurgit comme une forme particulière d'organisation des rapports sociaux au sein du champ scientifique.

La discipline, telle qu'on la découvre à travers l'habitus disciplinaire, rend inutile la distinction entre les conditions internes et externes d'élaboration des connaissances. La construction de la discipline est indissociable des jeux stratégiques toujours fluctuants que définissent les acteurs. La discipline, on l'aura compris, se structure et se déforme au rythme où se font et se défont les alliances entre les protagonistes eux-mêmes. 